University of Wollongong

Research Online

Faculty of Social Sciences - Papers (Archive) Faculty of Arts, Social Sciences \& Humanities

2017

Perceived public transport infrastructure modifies the association between public transport use and mental health: Multilevel analyses from the United Kingdom

Xiaoqi Feng

University of Wollongong, xfeng@uow.edu.au

Zhiqiang Feng

University of Edinburgh

Thomas E. Astell-Burt

University of Wollongong, thomasab@uow.edu.au

Follow this and additional works at: https://ro.uow.edu.au/sspapers

Part of the Education Commons, and the Social and Behavioral Sciences Commons

Research Online is the open access institutional repository for the University of Wollongong. For further information contact the UOW Library: research-pubs@uow.edu.au 


\title{
Perceived public transport infrastructure modifies the association between public transport use and mental health: Multilevel analyses from the United Kingdom
}

\author{
Abstract \\ Aims Investments to promote public transport utilisation are being championed to achieve sustainable \\ development, but the potential co-benefits for mental health are comparatively under-researched. We \\ hypothesised that frequent users of public transport would be more likely to have better mental health \\ (possibly due to increased levels of physical activity), but among the more frequent users, less favourable \\ perceptions of public transport infrastructure (PPTI) could have a negative influence on mental health. \\ Methods Multilevel linear and logistic regressions were fitted on 30,214 participants in the UK Household \\ Longitudinal Study with lagged PPTI and confounder measures at baseline and indicators of active travel \\ and mental health (General Health Questionnaire (GHQ), SF-12 Mental Component Scale (MCS) and the \\ Warwick Edinburgh Mental Well Being Scale (WEMWBS)) at follow-up. Results Compared to participants \\ expressing poor PPTI, those who felt it was excellent were $1.29(95 \% \mathrm{Cl} 1.15,1.45)$ times more likely to be \\ frequent users of public transport and $1.53(95 \% \mathrm{Cl} 1.33,1.76)$ times more likely to choose to walk or cycle \\ journeys of less than two to three miles. Frequent use of public transport was found to be consistently \\ associated with better mental health for GHQ caseness (OR $0.85,95 \% \mathrm{Cl} 0.79,0.91$ ), GHQ score \\ (coefficient $-0.28,95 \% \mathrm{Cl}-0.41,-0.16$ ), MCS (coefficient $0.45,95 \% \mathrm{Cl} 0.23,0.66$ ), and WEMWBS (coefficient \\ $0.30,95 \% \mathrm{Cl} 0.19,0.40)$. Among frequent users of public transport, participants expressing poor PPTI \\ were $1.46(95 \% \mathrm{Cl} 1.11,1.93)$ times more likely to report poorer mental health according to the GHQ \\ caseness indicator, compared to frequent users that regarded PPTI as excellent. Similar results were \\ observed for the other indicators of mental health. Conclusions These findings indicate that while the \\ provision of public transport infrastructure is a necessary pre-condition for stimulating population \\ increases in physical activity, PPTI improvements needs to be prioritised to leverage the full mental \\ health-related co-benefits of active travel. \\ Disciplines \\ Education | Social and Behavioral Sciences

\section{Publication Details} \\ Feng, X., Feng, Z. \& Astell-Burt, T. (2017). Perceived public transport infrastructure modifies the \\ association between public transport use and mental health: Multilevel analyses from the United \\ Kingdom. PLoS One, 12 (8), e0180081-1-e0180081-17.
}




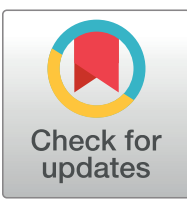

\section{G openaccess}

Citation: Feng X, Feng Z, Astell-Burt T (2017) Perceived public transport infrastructure modifies the association between public transport use and mental health: Multilevel analyses from the United Kingdom. PLoS ONE 12(8): e0180081. https://doi. org/10.1371/journal.pone.0180081

Editor: Ali Montazeri, Iranian Institute for Health Sciences Research, ISLAMIC REPUBLIC OF IRAN

Received: January 11, 2016

Accepted: June 10, 2017

Published: August 16, 2017

Copyright: ๑ 2017 Feng et al. This is an open access article distributed under the terms of the Creative Commons Attribution License, which permits unrestricted use, distribution, and reproduction in any medium, provided the original author and source are credited.

Data Availability Statement: Data owned by a non-author third party and are available through a process of application to the UK Data Archive for researchers who meet the criteria for access. The UK Data Archive is the lead organisation of the UK Data Service, which provides unified access to the UK's largest collection of social, economic and population data. The website for more information is: http://www.data-archive.ac.uk/.
RESEARCH ARTICLE

\section{Perceived public transport infrastructure modifies the association between public transport use and mental health: Multilevel analyses from the United Kingdom}

\author{
Xiaoqi Feng ${ }^{1,2 *}$, Zhiqiang Feng ${ }^{3}$, Thomas Astell-Burt ${ }^{1,2}$ \\ 1 Population Wellbeing and Environment Research Lab (PowerLab), School of Health and Society, Faculty of \\ Social Sciences, University of Wollongong, Wollongong, Australia, 2 Menzies Centre for Health Policy, \\ School of Public Health, University of Sydney, Sydney, Australia, 3 School of Geosciences, Drummond \\ Library, Surgeon's Square, Drummond Street, Edinburgh, Scotland, United Kingdom \\ *xfeng@uow.edu.au
}

\section{Abstract}

\section{Aims}

Investments to promote public transport utilisation are being championed to achieve sustainable development, but the potential co-benefits for mental health are comparatively under-researched. We hypothesised that frequent users of public transport would be more likely to have better mental health (possibly due to increased levels of physical activity), but among the more frequent users, less favourable perceptions of public transport infrastructure (PPTI) could have a negative influence on mental health.

\section{Methods}

Multilevel linear and logistic regressions were fitted on 30,214 participants in the UK Household Longitudinal Study with lagged PPTI and confounder measures at baseline and indicators of active travel and mental health (General Health Questionnaire (GHQ), SF-12 Mental Component Scale (MCS) and the Warwick Edinburgh Mental Well Being Scale (WEMWBS)) at follow-up.

\section{Results}

Compared to participants expressing poor PPTI, those who felt it was excellent were 1.29 $(95 \% \mathrm{Cl} 1.15,1.45)$ times more likely to be frequent users of public transport and $1.53(95 \%$ $\mathrm{Cl} 1.33,1.76)$ times more likely to choose to walk or cycle journeys of less than two to three miles. Frequent use of public transport was found to be consistently associated with better mental health for $\mathrm{GHQ}$ caseness (OR $0.85,95 \% \mathrm{Cl} 0.79,0.91$ ), GHQ score (coefficient $-0.28,95 \% \mathrm{Cl}-0.41,-0.16$ ), MCS (coefficient $0.45,95 \% \mathrm{Cl} 0.23,0.66$ ), and WEMWBS (coefficient $0.30,95 \% \mathrm{Cl} 0.19,0.40)$. Among frequent users of public transport, participants expressing poor PPTI were $1.46(95 \% \mathrm{Cl} 1.11,1.93)$ times more likely to report poorer mental health according to the GHQ caseness indicator, compared to frequent users that 
Funding: Dr Xiaoqi Feng's time on the project was partially funded by a postdoctoral fellowship with the National Heart Foundation of Australia.

Competing interests: The authors have declared that no competing interests exist. regarded PPTI as excellent. Similar results were observed for the other indicators of mental health.

\section{Conclusions}

These findings indicate that while the provision of public transport infrastructure is a necessary pre-condition for stimulating population increases in physical activity, PPTI improvements needs to be prioritised to leverage the full mental health-related co-benefits of active travel.

\section{Introduction}

Despite considerable investment in scientific research worldwide, there remains little evidence of interventions that promote physical activity in the medium-to-long term [1]. Up till relatively recently, this evidence has been largely focussed upon person-level factors and the role of practitioners, but now there is a wider appreciation that many decisions made outside the more traditional purview of the health sector are fundamental determinants of healthy, active and long lives [2]. If the environments that we design and build go on to shape where people live and what they can choose to do with their time [3], however, this suggests radical (i.e. population-level [4]) change is a necessary pre-condition to stimulate positive and sustained behavioural change $[5,6]$. It is in this context that the co-benefits of engendering active travel within daily life as a means for long-term improvements in physical activity and associated health status, such as the prevention of cardiometabolic diseases like type 2 diabetes mellitus, come to the fore [7-11].

Active travel generally equates to walking for transport and cycling, but also to the use of public transport, for which physical activity is a clear factor, unlike when travelling in cars. The determination of active travel via public transport is likely to be influenced, therefore, by a complex interplay between person-level characteristics and the availability of public transport infrastructure [12]. Journeys over short distances, for example, may be easily traversable by walking or cycling, but longer journeys usually invoke a choice to go by car or, if sufficient infrastructure is available, via public transport. It is the word 'sufficient' that is problematic, however, given that just because public transport infrastructure is available does not mean it is deemed to be of an acceptable standard for use. Perceptions of public transport that are likely to matter for whether a person considers it an option for active travel probably include a lack of information, high cost, if it is infrequent or unreliable, difficult to access, or perceived unsafe $[13,14]$.

Thus, while active travel via public transport may lead to gains in physical activity per se relative to travelling by car, if a person has little choice but to use public transport they perceive as of unacceptable quality, this may in fact be an important source of psychosocial stress that accumulates and repeatedly insults over time, perhaps resulting in loss of a sense of control over one's life, with negative downstream impacts on psychological and physiological health [15-17]. Research on this issue has been scant, but what evidence there is remains equivocal. A recent study, for example, reported benefits of active travel for physical health but null findings for mental health [18]. Meanwhile, previous research has suggested that the psychological implications of using public transport as opposed to cars may potentially include reduced feelings of autonomy, mastery, prestige and self-esteem [19]. These findings demonstrate that the relation between active travel and mental health is not straightforward, yet has clear health and 
policy relevance. Accordingly, the purpose of our study was to examine the interplay between perceptions of public transport infrastructure (PPTI, hereafter) and use of public transport for a range of mental health indicators.

\section{Method}

\section{Setting}

The study is set in the United Kingdom of Great Britain and Northern Island. The UK is an ideal setting for this research with just over half of all distances travelled as car drivers and about 27\% in addition as car passengers in 2010 [20]. Although there has been growth in use of trains, this has reportedly come from more people initiating travel by public transport rather than greater frequency of use among existing rail users [20]. This growth in demand has manifested after (and perhaps in spite of) the infamous privatisation of the UK rail network [21, 22]. Importantly, there are significant geographic variations in public transport availability, with rail travel more common car travel less common among residents in London compared to those outside the capital [20].

\section{Design}

PPTI was conceptualised as an antecedent of public transport use on the proviso that those who judge public transport to be of poor quality are likely to select alternative modes of transportation. PPTI was not considered to be a direct antecedent of mental health, however, it was considered to be an effect modifier of the potentially causal effect of public transport use on mental health. For the many people who do use public transport, this experience is hypothesised to be a variable source of psychosocial stress depending upon the level of PPTI. Public transport use, PPTI and mental health are all confounded by various indicators as illustrated.

\section{Data}

The data used for this study was an extract from the UK Household Longitudinal Study (UKHLS), for which information on the design, sampling and content are already available $[23,24]$. In brief, the UKHLS is a large panel study consisting of waves collected over two years or 24 months, with the first wave in beginning in 2009-10. Interviews with participants took place annually using computer assisted personal interviewing (CAPI), rendering a pattern of overlapping waves as part of the design. One household member completed the household enumeration grid and the household interview. Each resident of an enumerated household aged 16 years or older was administered an individual adult interview and a self-completed questionnaire. Questionnaire instruments and survey materials were translated into nine languages. The overall response rate at wave 1 was $57.3 \%(n=50,199)$, which is suggested to be typical for multi-purpose surveys of this type in the UK. The data extract used in this study was from waves 3 (2011-2012) and 4 (2012-2013), with response rates of $76.1 \%$ and $80.7 \%$ respectively,[24] due to the availability of relevant indicators for this study. Herein, data from wave 3 (PPTI and confounders) are referred to as 'baseline' whereas data from wave 4 (mental health and public transport use) is labelled 'follow-up'.

\section{Mental health}

Four contrasting indicators of mental health were examined at follow-up in order to triangulate the potential influence of PPTI and public transport use. The first two indicators were based upon a measure of minor psychiatric morbidity, derived from the 12-item General Health Questionnaire (GHQ) [25]. The GHQ-12 was developed as a screening instrument for 
use in primary care settings. It includes questions on concentration, sleep loss due to worry, perception of role, capability in decision making, whether constantly under strain, perception of problems in overcoming difficulties, enjoyment of day-to-day activities, coping resources, loss of confidence, self-worth, general happiness and whether suffering depression or unhappiness [26]. Responses to these items are summed to construct a continuous measure. The first mental health indicator was a binary variable denoting participants with GHQ scores of 4 or greater were classified as having clinically significant minor psychiatric morbidity, compared with those scoring less than 4 . This threshold has been identified as appropriate within the UK population [27]. The second indicator of mental health was the GHQ-12 score, a normally-distributed (i.e. continuous) variable that is derived from the same questions as the GHQ-12 [25, 26].

The third indicator of mental health used in this study was the mental component scale (MCS) of the 12-item Short Form Health Survey (SF-12), a commonly used measure of general health and functioning in epidemiological research [24]. The SF-12 is an abbreviated form of the 36-item instrument (SF-36) designed to assess general self-rated health, physical and psychological symptoms, and limitations in everyday activity due to physical and mental health over the previous 4 weeks [28]. The MCS was developed using item weights based on an orthogonal factor rotation and scores have been used to successfully discriminate between the presence and severity of mental disorders in clinically defined groups of adults, though there is no widely accepted screening cut-off score for probable diagnosis of any mental disorder [29]. As such, the MCS was used as a continuous outcome variable with normal distribution in this study.

The fourth indicator of mental health used was the Warwick Edinburgh Mental Well Being Scale (WEMWBS) [30]. Whereas the GHQ and SF-12-based MCS are indicators that focus upon negative aspects of mental health, WEMWBS provides a counterpoint by emphasising positive mental health (a term often used interchangeably with 'mental wellbeing'). WEMWBS attempts to capture affective-emotional aspects, cognitive-evaluative dimensions and psychological functioning by focussing entirely on the positive and, therefore, free of so-called 'ceiling effects'. WEMWBS has been shown to have high levels of internal consistency and reliability against accepted criteria [31]. In this study, WEMWBS follows a normal distribution and is used as a continuous outcome variable.

\section{Active travel}

Two indicators of active travel were obtained at follow-up. Participants were asked 'could you tell me how often you personally': (i) 'use public transport (e.g. bus, train) rather than travel by car?'; (ii) 'walk or cycle for short journeys less than 2 or 3 miles?' We constructed binary variables for each, distinguishing between participants in active travel responding 'always', 'very often' or 'quite often' (referred to hereafter as 'frequent'), in comparison to those responding 'not very often' or 'never' (i.e. 'infrequent').

\section{Perceptions of public transport infrastructure (PPTI)}

PPTI indicators were only available at baseline. All participants were asked 'How would you rate public transport services in your local area?' Participants had the option to rate public transport as 'excellent', 'very good', 'fair', 'poor', or 'no opinion'.

\section{Confounders}

A range of demographics (age, gender), socioeconomic factors (highest educational qualification and economic status e.g. employment, retired), physical functioning, and urban/rural 
status of the area of residence were taken into account as potential confounders of public transport availability and mental health. These confounders were all measured at baseline, to avoid the potential for reverse causation with public transport use and mental health measured at follow-up.

\section{Analysis}

The data extract was restricted to participants from baseline $(n=54,781)$ who were successfully followed up $(\mathrm{n}=42,115)$ and did not move home in between $(\mathrm{n}=35,931)$. This sampling strategy was implemented to minimise the likelihood of a change in provision of local public transport, so that the PPTI variable measured at baseline could be expected to be consistent also at follow-up. This sample was further reduced to those participants with full mental health data for each of the four indicators $(n=30,214)$. Logistic regression was used to examine the patterning of missing mental health outcome data against PPTI, active travel and the aforementioned confounders.

A three-level multilevel model was employed to account for the nesting of persons (level 1, $\mathrm{n}=30,214$ ) within households (level $2, \mathrm{n}=19,528$ ) due to the nature of the UKHLS. Regional variation, such as the differences in public transport infrastructure within London compared with other areas of the UK, were also taken into account (level 3, $\mathrm{n}=12$ Government Office Regions). An average of 1.5 participants were clustered within households $(\min =1, \max =7)$, whereas the equivalent average for regions was $2517.8(\min =1161$, $\max =3685)$. Multilevel logistic regression was used to analyse the patterning of poor PPTI across each of the confounding variables. The same regression technique was then applied to assess the degree of association between PPTI and active travel one year later, adjusting for the confounders. Multilevel logistic and linear regressions were then used to examine the interplay of PPTI and public transport use for predicting each of the four mental health outcomes (logistic regression for GHQ-12 caseness, linear regression for the GHQ-12 score, MCS and WEMWBS). In each of these models, the variance partition coefficient and, where relevant, the median odds ratio were calculated to report variation in each outcome across households and regions [32]. All models were run in MLwIN v.2.31 [33].

\section{Results}

The odds of missing mental health indicator data were lower among women than men $(\mathrm{OR}=0.92,95 \% \mathrm{CI} 0.86,0.97)$, higher among older participants (e.g. $\geq 70 \mathrm{y}$ compared with 15 $19 \mathrm{y} \mathrm{OR}=2.78,95 \% \mathrm{CI} 2.40,3.22$ ), higher among participants without qualifications $(\mathrm{OR}=4.29,95 \% \mathrm{CI} 5.58,7.90)$, higher among the unemployed ( $\mathrm{OR}=1.81,95 \% \mathrm{CI} 1.59,2.07)$, the retired $(\mathrm{OR}=2.14,95 \% \mathrm{CI} 2.00,2.28)$, the long-term sick or disabled $(\mathrm{OR}=2.64,95 \% \mathrm{CI}$ $2.31,3.01$ ), those reporting more favourable PPTI (e.g. excellent versus poor $\mathrm{OR}=1.21,95 \% \mathrm{CI}$ $1.07,1.37)$, and participants who often chose to use public transport $(\mathrm{OR}=1.37,95 \% \mathrm{CI} 1.28$, 1.47). Missing data was less common among participants with higher physical functioning (e.g. highest versus lowest quintile $\mathrm{OR}=0.018,95 \% \mathrm{CI} 0.016,0.021$ ), living in rural areas compared with urban $(\mathrm{OR}=0.80,95 \% \mathrm{CI} 0.75,0.85)$, and who chose to walk or cycle short journeys less than two to three miles ( $\mathrm{OR}=0.86,95 \% \mathrm{CI} 0.81,0.92)$.

Descriptive findings concerning the patterning of poorer mental health with respect to sociodemographic characteristics were in line with previous literature [34, 35]. Poorer mental health was observed among women compared with men across all four indicators (Table 1). Mental health was also found to vary in a curvilinear fashion by age, with poorer levels observed among younger to middle-aged adults. Participants with lower levels of education, the unemployed and retired, those with lower physical functioning and those living in urban 
Table 1. Descriptive statistics and patterning of mental health variables.

\begin{tabular}{|c|c|c|c|c|c|c|}
\hline & \multirow[t]{2}{*}{$\mathbf{N}$} & \multirow[t]{2}{*}{$\%$} & \multirow{2}{*}{$\begin{array}{l}\text { GHQ caseness } \\
\% \geq 4(95 \% \mathrm{Cl})\end{array}$} & \multirow{2}{*}{$\begin{array}{c}\text { GHQ score } \\
\text { Mean }(95 \% \mathrm{Cl})\end{array}$} & \multirow{2}{*}{$\begin{array}{c}\text { MCS } \\
\text { Mean }(95 \% \mathrm{Cl})\end{array}$} & \multirow{2}{*}{$\begin{array}{c}\text { WEMWBS } \\
\text { Mean }(95 \% \mathrm{Cl})\end{array}$} \\
\hline & & & & & & \\
\hline \multicolumn{7}{|l|}{ Gender } \\
\hline male & 13,127 & 43.4 & $\begin{array}{c}14.57(13.98 \\
15.19)\end{array}$ & $\begin{array}{c}10.29(10.20 \\
10.38)\end{array}$ & $\begin{array}{c}50.78(50.61 \\
50.94)\end{array}$ & $\begin{array}{c}24.88(24.80 \\
24.95)\end{array}$ \\
\hline female & 17,090 & 56.6 & $\begin{array}{c}20.63(20.03 \\
21.25)\end{array}$ & $\begin{array}{c}11.44(11.36 \\
11.53)\end{array}$ & $\begin{array}{c}48.84(48.69 \\
48.98)\end{array}$ & $\begin{array}{c}24.62(24.55 \\
24.68)\end{array}$ \\
\hline \multicolumn{7}{|l|}{ Age group } \\
\hline $16-19 y$ & 1,702 & 5.6 & $\begin{array}{c}19.86(18.03 \\
21.82)\end{array}$ & $\begin{array}{c}10.40(10.15 \\
10.66)\end{array}$ & $\begin{array}{c}48.98(48.53 \\
49.43)\end{array}$ & $\begin{array}{c}24.32(24.11 \\
24.53)\end{array}$ \\
\hline $20-24 y$ & 1,406 & 4.7 & $\begin{array}{c}20.84(18.80 \\
23.04)\end{array}$ & $\begin{array}{c}11.11(10.83 \\
11.39)\end{array}$ & $\begin{array}{c}48.14(47.64 \\
48.63)\end{array}$ & $\begin{array}{c}24.02(23.78 \\
24.25)\end{array}$ \\
\hline $25-29 y$ & 1,735 & 5.7 & $\begin{array}{c}20.29(18.46 \\
22.25)\end{array}$ & $\begin{array}{c}11.16(10.91 \\
11.41)\end{array}$ & $\begin{array}{c}47.56(47.11 \\
48.00)\end{array}$ & $\begin{array}{c}24.02(23.81 \\
24.23)\end{array}$ \\
\hline $30-34 y$ & 2,348 & 7.8 & $\begin{array}{c}19.63(18.08 \\
21.29)\end{array}$ & $\begin{array}{c}11.12(10.90 \\
11.33)\end{array}$ & $\begin{array}{c}47.98(47.59 \\
48.36)\end{array}$ & $\begin{array}{c}24.38(24.20 \\
24.56)\end{array}$ \\
\hline $35-39 y$ & 2,655 & 8.8 & $\begin{array}{c}19.17(17.72 \\
20.71)\end{array}$ & $\begin{array}{c}11.19(10.99 \\
11.39)\end{array}$ & $\begin{array}{c}48.27(47.90 \\
48.63)\end{array}$ & $\begin{array}{c}24.43(24.26 \\
24.60)\end{array}$ \\
\hline $40-44 y$ & 3,166 & 10.5 & $\begin{array}{c}19.49(18.15 \\
20.91)\end{array}$ & $\begin{array}{c}11.36(11.17 \\
11.55)\end{array}$ & $\begin{array}{c}48.43(48.10 \\
48.76)\end{array}$ & $\begin{array}{c}24.41(24.26 \\
24.56)\end{array}$ \\
\hline $45-49 y$ & 3,036 & 10.1 & $\begin{array}{c}19.76(18.38 \\
21.22)\end{array}$ & $\begin{array}{c}11.52(11.33 \\
11.71)\end{array}$ & $\begin{array}{c}49.09(48.75 \\
49.42)\end{array}$ & $\begin{array}{c}24.35(24.19 \\
24.51)\end{array}$ \\
\hline $50-54 y$ & 2,896 & 9.6 & $\begin{array}{c}21.72(20.26 \\
23.26)\end{array}$ & $\begin{array}{c}11.68(11.48 \\
11.87)\end{array}$ & $\begin{array}{c}48.98(48.63 \\
49.32)\end{array}$ & $\begin{array}{c}24.32(24.16 \\
24.48)\end{array}$ \\
\hline $55-59 y$ & 2,545 & 8.4 & $\begin{array}{c}19.17(17.69 \\
20.75)\end{array}$ & $\begin{array}{c}11.32(11.11 \\
11.53)\end{array}$ & $\begin{array}{c}49.84(49.47 \\
50.21)\end{array}$ & $\begin{array}{c}24.75(24.58 \\
24.92) \\
\end{array}$ \\
\hline $60-64 y$ & 2,637 & 8.7 & $\begin{array}{c}14.52(13.23 \\
15.92)\end{array}$ & $\begin{array}{c}10.42(10.21 \\
10.62)\end{array}$ & $\begin{array}{c}51.54(51.17 \\
51.90)\end{array}$ & $\begin{array}{c}25.49(25.32 \\
25.65)\end{array}$ \\
\hline $65-69 y$ & 2,345 & 7.8 & $\begin{array}{c}12.37(11.09 \\
13.76)\end{array}$ & $\begin{array}{c}10.01(9.80 \\
10.23)\end{array}$ & $\begin{array}{c}52.70(52.32 \\
53.09)\end{array}$ & $\begin{array}{c}25.90(25.72 \\
26.08)\end{array}$ \\
\hline $70 y$ or older & 3,746 & 12.4 & $\begin{array}{c}12.79(11.76 \\
13.90)\end{array}$ & $\begin{array}{c}10.05(9.88 \\
10.22)\end{array}$ & $\begin{array}{c}52.40(52.10 \\
52.71)\end{array}$ & $\begin{array}{c}25.56(25.42 \\
25.70)\end{array}$ \\
\hline \multicolumn{7}{|c|}{ Highest educational qualifications } \\
\hline Degree & 7,143 & 23.6 & $\begin{array}{c}16.39(15.55 \\
17.27)\end{array}$ & $\begin{array}{c}10.61(10.48 \\
10.73)\end{array}$ & $\begin{array}{c}49.91(49.69 \\
50.14)\end{array}$ & $\begin{array}{c}25.41(25.31 \\
25.52)\end{array}$ \\
\hline Other higher degree & 3,590 & 11.9 & $\begin{array}{c}16.94(15.74 \\
18.20)\end{array}$ & $\begin{array}{c}10.83(10.65 \\
11.01)\end{array}$ & $\begin{array}{c}50.08(49.76 \\
50.39)\end{array}$ & $\begin{array}{c}25.04(24.89 \\
25.18)\end{array}$ \\
\hline A-level & 6,094 & 20.2 & $\begin{array}{c}17.77(16.83 \\
18.75)\end{array}$ & $\begin{array}{c}10.86(10.73 \\
11.00)\end{array}$ & $\begin{array}{c}49.58(49.34 \\
49.82)\end{array}$ & $\begin{array}{c}24.69(24.57 \\
24.80)\end{array}$ \\
\hline GCSE & 6,495 & 21.5 & $\begin{array}{c}18.89(17.96 \\
19.86)\end{array}$ & $\begin{array}{c}11.09(10.96 \\
11.22)\end{array}$ & $\begin{array}{c}49.36(49.12 \\
49.59)\end{array}$ & $\begin{array}{c}24.26(24.15 \\
24.37) \\
\end{array}$ \\
\hline Other qualification & 2,900 & 9.6 & $\begin{array}{c}18.79(17.41 \\
20.26)\end{array}$ & $\begin{array}{c}11.15(10.95 \\
11.34)\end{array}$ & $\begin{array}{c}50.13(49.78 \\
50.48)\end{array}$ & $\begin{array}{c}24.36(24.20 \\
24.52)\end{array}$ \\
\hline No qualification & 3,624 & 12.0 & $\begin{array}{c}20.28(19.00 \\
21.62)\end{array}$ & $\begin{array}{c}11.45(11.27 \\
11.62)\end{array}$ & $\begin{array}{c}49.30(48.99 \\
49.62)\end{array}$ & $\begin{array}{c}24.33(24.18 \\
24.47)\end{array}$ \\
\hline Missing & 371 & 1.2 & $\begin{array}{c}18.87(15.20 \\
23.17)\end{array}$ & $\begin{array}{c}10.75(10.20 \\
11.29)\end{array}$ & $\begin{array}{c}48.81(47.83 \\
49.79)\end{array}$ & $\begin{array}{c}24.32(23.87 \\
24.77)\end{array}$ \\
\hline \multicolumn{7}{|l|}{ Economic status } \\
\hline employed & 17,377 & 57.5 & $\begin{array}{c}16.03(15.49 \\
16.59)\end{array}$ & $\begin{array}{c}10.63(10.56 \\
10.71)\end{array}$ & $\begin{array}{c}49.93(49.80 \\
50.07)\end{array}$ & $\begin{array}{c}24.87(24.81 \\
24.94)\end{array}$ \\
\hline unemployed & 1,306 & 4.3 & $\begin{array}{c}30.09(27.66 \\
32.64)\end{array}$ & $\begin{array}{c}12.90(12.62 \\
13.18)\end{array}$ & $\begin{array}{c}46.02(45.52 \\
46.52)\end{array}$ & $\begin{array}{c}22.66(22.43 \\
22.90)\end{array}$ \\
\hline retired & 7,496 & 24.8 & $\begin{array}{c}13.78(13.02 \\
14.58)\end{array}$ & $\begin{array}{c}10.23(10.11 \\
10.34)\end{array}$ & $\begin{array}{c}52.07(51.86 \\
52.28)\end{array}$ & $\begin{array}{c}25.61(25.51 \\
25.70)\end{array}$ \\
\hline
\end{tabular}

(Continued) 
Table 1. (Continued)

\begin{tabular}{|c|c|c|c|c|c|c|}
\hline & \multirow[t]{2}{*}{$\mathbf{N}$} & \multirow[t]{2}{*}{$\%$} & GHQ caseness & \multirow{2}{*}{$\begin{array}{c}\text { GHQ score } \\
\text { Mean }(95 \% \mathrm{Cl})\end{array}$} & \multirow{2}{*}{$\begin{array}{c}\text { MCS } \\
\text { Mean }(95 \% \mathrm{Cl})\end{array}$} & \multirow{2}{*}{$\begin{array}{c}\text { WEMWBS } \\
\text { Mean }(95 \% \mathrm{CI})\end{array}$} \\
\hline & & & $\% \geq 4(95 \% \mathrm{Cl})$ & & & \\
\hline family care & 1,593 & 5.3 & $\begin{array}{c}25.30(23.22 \\
27.49)\end{array}$ & $\begin{array}{c}12.31(12.05 \\
12.56)\end{array}$ & $\begin{array}{c}46.70(46.25 \\
47.16)\end{array}$ & $\begin{array}{c}23.78(23.57 \\
23.99)\end{array}$ \\
\hline student & 1,363 & 4.5 & $\begin{array}{c}19.96(17.92 \\
22.16)\end{array}$ & $\begin{array}{c}10.70(10.42 \\
10.98)\end{array}$ & $\begin{array}{c}48.52(48.02 \\
49.01)\end{array}$ & $\begin{array}{c}24.52(24.29 \\
24.75)\end{array}$ \\
\hline sick/disabled & 956 & 3.2 & $\begin{array}{c}54.39(51.22 \\
57.53)\end{array}$ & $\begin{array}{c}17.41(17.07 \\
17.74)\end{array}$ & $\begin{array}{c}38.17(37.58 \\
38.76)\end{array}$ & $\begin{array}{c}20.09(19.82 \\
20.37)\end{array}$ \\
\hline don't know & 126 & 0.4 & $\begin{array}{c}25.40(18.56 \\
33.71)\end{array}$ & $\begin{array}{c}12.09(11.18 \\
13.00)\end{array}$ & $\begin{array}{c}47.50(45.88 \\
49.11)\end{array}$ & $\begin{array}{c}23.84(23.08 \\
24.60)\end{array}$ \\
\hline \multicolumn{7}{|l|}{ Physical functioning (quintiles) } \\
\hline 1 (low) & 2,686 & 8.9 & $\begin{array}{c}38.38(36.56 \\
40.24)\end{array}$ & $\begin{array}{c}14.45(14.25 \\
14.64)\end{array}$ & $\begin{array}{c}47.29(46.94 \\
47.63)\end{array}$ & $\begin{array}{c}22.92(22.76 \\
23.09)\end{array}$ \\
\hline 2 & 6,755 & 22.4 & $\begin{array}{c}21.20(20.24 \\
22.19)\end{array}$ & $\begin{array}{c}11.64(11.52 \\
11.76)\end{array}$ & $\begin{array}{c}50.37(50.16 \\
50.59)\end{array}$ & $\begin{array}{c}24.17(24.07 \\
24.28)\end{array}$ \\
\hline 3 & 7,018 & 23.2 & $\begin{array}{c}13.78(12.99 \\
14.61)\end{array}$ & $\begin{array}{c}10.24(10.12 \\
10.36)\end{array}$ & $\begin{array}{c}50.82(50.61 \\
51.04)\end{array}$ & $\begin{array}{c}24.87(24.77, \\
24.97)\end{array}$ \\
\hline 4 & 7,648 & 25.3 & $7.37(6.81,7.98)$ & $8.94(8.82,9.05)$ & $\begin{array}{c}52.89(52.69 \\
53.10)\end{array}$ & $\begin{array}{c}25.97(25.87 \\
26.07)\end{array}$ \\
\hline 5 (high) & 6,110 & 20.2 & $\begin{array}{c}23.65(22.60 \\
24.73)\end{array}$ & $\begin{array}{c}11.96(11.83 \\
12.08)\end{array}$ & $\begin{array}{c}44.63(44.40 \\
44.86)\end{array}$ & $\begin{array}{c}24.42(24.31 \\
24.53)\end{array}$ \\
\hline \multicolumn{7}{|l|}{ Urban/rural } \\
\hline urban & 22,156 & 73.3 & $\begin{array}{c}18.85(18.34 \\
19.37)\end{array}$ & $\begin{array}{c}11.07(11.00 \\
11.14)\end{array}$ & $\begin{array}{c}49.29(49.16 \\
49.42)\end{array}$ & $\begin{array}{c}24.61(24.55 \\
24.67)\end{array}$ \\
\hline rural & 8,058 & 26.7 & $\begin{array}{c}15.66(14.88 \\
16.47)\end{array}$ & $\begin{array}{c}10.60(10.49 \\
10.72)\end{array}$ & $\begin{array}{c}50.75(50.54 \\
50.96)\end{array}$ & $\begin{array}{c}25.05(24.95 \\
25.15)\end{array}$ \\
\hline missing & 3 & 0.0 & $\begin{array}{c}33.33(4.34 \\
84.65)\end{array}$ & $\begin{array}{c}11.67(5.57 \\
17.76)\end{array}$ & $\begin{array}{c}53.56(42.70 \\
64.42)\end{array}$ & $\begin{array}{c}28.00(22.96 \\
33.04)\end{array}$ \\
\hline \multicolumn{7}{|c|}{ Perceptions of public transport infrastructure } \\
\hline Poor & 3,606 & 11.9 & $\begin{array}{c}21.33(20.02 \\
22.69)\end{array}$ & $\begin{array}{c}11.50(11.32 \\
11.67)\end{array}$ & $\begin{array}{c}49.22(48.91 \\
49.54)\end{array}$ & $\begin{array}{c}24.69(24.54 \\
24.83)\end{array}$ \\
\hline Fair & 7,552 & 25.0 & $\begin{array}{c}18.33(17.47 \\
19.22)\end{array}$ & $\begin{array}{c}11.07(10.95 \\
11.19)\end{array}$ & $\begin{array}{c}49.33(49.11 \\
49.55)\end{array}$ & $\begin{array}{c}24.44(24.34 \\
24.54)\end{array}$ \\
\hline Very good & 12,334 & 40.8 & $\begin{array}{c}16.98(16.33 \\
17.65)\end{array}$ & $\begin{array}{c}10.76(10.66 \\
10.85)\end{array}$ & $\begin{array}{c}49.90(49.73 \\
50.07)\end{array}$ & $\begin{array}{c}24.80(24.72, \\
24.88)\end{array}$ \\
\hline Excellent & 3,384 & 11.2 & $\begin{array}{c}18.17(16.91 \\
19.51)\end{array}$ & $\begin{array}{c}10.67(10.49 \\
10.85)\end{array}$ & $\begin{array}{c}49.81(49.48 \\
50.13)\end{array}$ & $\begin{array}{c}25.12(24.97 \\
25.27)\end{array}$ \\
\hline No opinion & 3,015 & 10.0 & $\begin{array}{c}17.08(15.78 \\
18.47)\end{array}$ & $\begin{array}{c}11.00(10.81 \\
11.19)\end{array}$ & $\begin{array}{c}50.06(49.72 \\
50.41)\end{array}$ & $\begin{array}{c}24.79(24.63, \\
24.95)\end{array}$ \\
\hline Don't know/missing/proxy & 326 & 1.1 & $\begin{array}{c}19.02(15.12 \\
23.65)\end{array}$ & $\begin{array}{c}11.10(10.52 \\
11.69)\end{array}$ & $\begin{array}{c}49.53(48.49 \\
50.57)\end{array}$ & $\begin{array}{c}24.67(24.19 \\
25.16)\end{array}$ \\
\hline \multicolumn{7}{|c|}{$\begin{array}{l}\text { Choose to walk or cycle for short journeys less than } 2 \text { or } \\
3 \text { miles }\end{array}$} \\
\hline not often or never & 18,258 & 60.4 & $\begin{array}{l}18.01(17.46 \\
18.58)\end{array}$ & $\begin{array}{l}10.99(10.91 \\
11.07)\end{array}$ & $\begin{array}{l}49.76(49.62 \\
49.90)\end{array}$ & $\begin{array}{l}24.68(24.62 \\
24.75)\end{array}$ \\
\hline very often or more & 10,567 & 35.0 & $\begin{array}{l}16.17(15.48 \\
16.89)\end{array}$ & $\begin{array}{l}10.57(10.47 \\
10.67)\end{array}$ & $\begin{array}{l}49.91(49.73 \\
50.09)\end{array}$ & $\begin{array}{l}24.99(24.90 \\
25.07)\end{array}$ \\
\hline missing & 1,392 & 4.6 & $\begin{array}{l}31.68(29.29 \\
34.17)\end{array}$ & $\begin{array}{l}13.19(12.91 \\
13.47)\end{array}$ & $\begin{array}{l}46.91(46.40 \\
47.41)\end{array}$ & $\begin{array}{l}23.36(23.13 \\
23.59)\end{array}$ \\
\hline \multicolumn{7}{|c|}{ Choose to use public transport rather than car } \\
\hline not often or never & 23,186 & 76.7 & $\begin{array}{l}16.93(16.46 \\
17.42)\end{array}$ & $\begin{array}{l}10.82(10.75 \\
10.89)\end{array}$ & $\begin{array}{l}50.00(49.87 \\
50.12)\end{array}$ & $\begin{array}{l}24.83(24.77 \\
24.88)\end{array}$ \\
\hline very often or more & 5,380 & 17.8 & $\begin{array}{l}20.43(19.37 \\
21.53)\end{array}$ & $\begin{array}{l}11.10(10.96 \\
11.25)\end{array}$ & $\begin{array}{l}48.93(48.67 \\
49.19)\end{array}$ & $\begin{array}{l}24.49(24.38 \\
24.61)\end{array}$ \\
\hline
\end{tabular}

(Continued) 
Table 1. (Continued)

\begin{tabular}{|c|c|c|c|c|c|c|}
\hline & \multirow[t]{2}{*}{$\mathbf{N}$} & \multirow[t]{2}{*}{$\%$} & GHQ caseness & GHQ score & MCS & WEMWBS \\
\hline & & & $\% \geq 4(95 \% \mathrm{Cl})$ & Mean $(95 \% \mathrm{Cl})$ & Mean $(95 \% \mathrm{Cl})$ & Mean $(95 \% \mathrm{Cl})$ \\
\hline missing & 1,651 & 5.5 & $\begin{array}{c}25.08(23.04 \\
27.22)\end{array}$ & $\begin{array}{c}12.18(11.92 \\
12.44) \\
\end{array}$ & $\begin{array}{c}47.65(47.18 \\
48.11)\end{array}$ & $\begin{array}{c}24.12(23.91 \\
24.34)\end{array}$ \\
\hline
\end{tabular}

$\mathrm{GHQ}=$ General Health Questionnaire $\mid \mathrm{MCS}=\mathrm{SF}-12$ Mental Health Component $\mid$ WEMWBS = Warwick Edinburgh Mental Well Being Scale

areas also tended to have poorer mental health. Participants who often chose to walk or cycle for short journeys of less than two to three miles tended to have better mental health than those who did not. In contrast, participants who often chose to use public transport rather than a car tended to have poorer mental health, especially demonstrable with the GHQ caseness variable. These findings are broadly in line with those more generally that suggest higher levels of physical activity are associated with more favourable levels of mental health (e.g. [36]). New findings from this descriptive analysis suggest that participants with better PPTI tended to have better mental health, most clearly observable using the GHQ caseness indicator.

The odds of reporting poor PPTI were significantly higher among women than men, but did not appear to vary significantly by age or economic status (Table 2). Compared to participants with degrees, those with non-university educational qualifications were less likely to report poor PPTI. Participants with higher levels of physical functioning were significantly less likely to report poor PPTI, whereas those in rural areas with over four times more likely to report poor PPTI than their urban counterparts. PPTI was found to vary both regionally $(\mathrm{VPC}=1.3 \%)$ and, especially, at the household level $(\mathrm{VPC}=27.4 \%)$.

The age and gender adjusted odds of choosing to use public transport over a car often, and also to walk or cycle short journeys of less than two to three miles were higher among participants reporting more favourable PPTI (Table 3). This association was partially attenuated, but remained notable after adjusting for socioeconomic confounders, physical functioning and urban/rural variable. These results can also be seen in Fig 1. Contrasts were observed in the variances of each outcome variable. Whereas $1.3 \%$ of variation in frequent use of public transport manifested regionally and $9.1 \%$ at the household scale, the equivalent for choosing to walk or cycle short journeys was $8.7 \%$ between regions and $11.9 \%$ between households. These regional variations were only partially explained by adjustment for socioeconomic factors, physical functioning and urban/rural, with rural dwellers less likely to be active travellers for both outcomes.

In fully-adjusted models, frequent use of public transport was found to be consistently associated with better mental health for GHQ caseness (OR 0.85, 95\%CI 0.79, 0.91), GHQ-36 (coefficient $-0.28,95 \% \mathrm{CI}-0.41,-0.16$ ), MCS (coefficient $0.45,95 \% \mathrm{CI} 0.23,0.66$ ), and WEMWBS (coefficient $0.30,95 \% \mathrm{CI} 0.19,0.40$ ). Frequent selection of walking or cycling for journeys of distances less than two to three miles was not associated with GHQ caseness (OR $1.11,95 \% \mathrm{CI} 0.16,7.96$ ) and GHQ-36 (coefficient $0.14,95 \% \mathrm{CI}-0.02,0.29$ ), but it was associated with lower levels (i.e. less favourable) of MCS (coefficient $-0.60,95 \% \mathrm{CI}-0.85,-0.29$ ), and WEMWBS (coefficient $-0.17,95 \% \mathrm{CI}-0.31,-0.04$ ).

The focus in the final set of analyses focussed on the effect modification of observed benefit of public transport use on mental health by PPTI. The fully-adjusted cross-classification of PPTI and use of public transport was associated with poorer mental health in the hypothesised direction (Table 4). Among frequent users of public transport, participants who regarded PPTI as of poor quality were 1.46 times more likely to report poorer mental health according to the GHQ caseness indicator $(95 \% \mathrm{CI} 1.11,1.93)$, compared to frequent users that regarded 
Table 2. Patterning of poor perceptions of public transport infrastructure.

\begin{tabular}{|c|c|}
\hline Fixed Part & OR (95\%Cl) \\
\hline \multicolumn{2}{|l|}{ Gender (ref: male) } \\
\hline female & $1.10(1.02,1.19)$ \\
\hline \multicolumn{2}{|l|}{ Age group (16-19y) } \\
\hline $20-24 y$ & $1.21(0.92,1.58)$ \\
\hline $25-29 y$ & $0.84(0.63,1.12)$ \\
\hline $30-34 y$ & $0.73(0.55,0.97)$ \\
\hline $35-39 y$ & $0.82(0.62,1.07)$ \\
\hline $40-44 y$ & $0.83(0.64,1.08)$ \\
\hline $45-49 y$ & $1.03(0.79,1.34)$ \\
\hline $50-54 y$ & $1.03(0.79,1.33)$ \\
\hline $55-59 y$ & $1.26(0.97,1.64)$ \\
\hline $60-64 y$ & $1.03(0.78,1.37)$ \\
\hline $65-69 y$ & $0.92(0.68,1.26)$ \\
\hline $70 y$ or older & $0.86(0.63,1.17)$ \\
\hline \multicolumn{2}{|c|}{ Highest educational qualification (ref: Degree) } \\
\hline Other higher degree & $0.92(0.81,1.06)$ \\
\hline A-level & $0.85(0.76,0.96)$ \\
\hline GCSE & $0.81(0.72,0.91)$ \\
\hline Other qualification & $0.78(0.67,0.91)$ \\
\hline No qualification & $0.73(0.63,0.85)$ \\
\hline Missing & $1.00(0.70,1.43)$ \\
\hline \multicolumn{2}{|l|}{ Economic status (ref: employed) } \\
\hline unemployed & $1.14(0.93,1.39)$ \\
\hline retired & $0.84(0.71,0.99)$ \\
\hline family care & $0.91(0.75,1.10)$ \\
\hline student & $0.86(0.66,1.12)$ \\
\hline sick/disabled & $0.89(0.70,1.13)$ \\
\hline don’t know & $1.13(0.64,2.00)$ \\
\hline \multicolumn{2}{|l|}{ Physical functioning (ref: 1 (low)) } \\
\hline 2 & $0.79(0.68,0.93)$ \\
\hline 3 & $0.72(0.61,0.85)$ \\
\hline 4 & $0.63(0.54,0.75)$ \\
\hline 5 (high) & $0.73(0.61,0.86)$ \\
\hline \multicolumn{2}{|l|}{ Urban/rural (ref: urban) } \\
\hline rural & $4.49(4.10,4.90)$ \\
\hline \multicolumn{2}{|l|}{ Random Part } \\
\hline Regional variance (standard error) & $0.061(0.027)$ \\
\hline VPC, MOR & $1.3 \%, 1.27$ \\
\hline Household variance (standard error) & $1.267(0.074)$ \\
\hline VPC, MOR & $27.4 \%, 2.93$ \\
\hline
\end{tabular}

https://doi.org/10.1371/journal.pone.0180081.t002

PPTI as excellent. This result was replicated across each of the other three indicators of mental health. Substantially weaker associations with mental health indicators were observed for participants expressing poor PPTI who were infrequent users of public transport. Predictions from these fully adjusted models are illustrated in Fig 2. A 5.89 percentage point gap in the 
Table 3. Association between public transport use and perceptions of public transport infrastructure.

\begin{tabular}{|c|c|c|c|c|}
\hline & \multicolumn{2}{|c|}{ Frequent user of public transport } & \multicolumn{2}{|c|}{ Frequently chooses to walk or cycle short journeys } \\
\hline & Model 1 & Model 2 & Model 1 & Model 2 \\
\hline Fixed Part & \multicolumn{4}{|c|}{ Odds Ratio (95\% Confidence Interval) } \\
\hline \multicolumn{5}{|l|}{ Gender (ref: male) } \\
\hline female & $0.86(0.82,0.91)$ & $0.86(0.81,0.90)$ & $1.19(1.12,1.27)$ & $1.18(1.11,1.27)$ \\
\hline \multicolumn{5}{|l|}{ Age group (16-19y) } \\
\hline $20-24 y$ & $0.70(0.60,0.81)$ & $0.80(0.67,0.96)$ & $0.67(0.56,0.79)$ & $0.94(0.77,1.14)$ \\
\hline $25-29 y$ & $0.68(0.59,0.79)$ & $0.83(0.70,0.99)$ & $0.45(0.38,0.54)$ & $0.71(0.58,0.87)$ \\
\hline $30-34 y$ & $0.66(0.57,0.75)$ & $0.83(0.70,0.98)$ & $0.29(0.24,0.34)$ & $0.46(0.37,0.57)$ \\
\hline $35-39 y$ & $0.62(0.54,0.71)$ & $0.81(0.68,0.96)$ & $0.31(0.27,0.37)$ & $0.51(0.42,0.62)$ \\
\hline $40-44 y$ & $0.53(0.46,0.60)$ & $0.69(0.59,0.82)$ & $0.26(0.22,0.30)$ & $0.41(0.33,0.50)$ \\
\hline $45-49 y$ & $0.52(0.46,0.59)$ & $0.71(0.60,0.84)$ & $0.25(0.21,0.29)$ & $0.40(0.33,0.49)$ \\
\hline $50-54 y$ & $0.54(0.48,0.62)$ & $0.75(0.63,0.89)$ & $0.30(0.26,0.35)$ & $0.46(0.38,0.57)$ \\
\hline $55-59 y$ & $0.61(0.53,0.70)$ & $0.84(0.71,1.00)$ & $0.30(0.26,0.36)$ & $0.45(0.36,0.55)$ \\
\hline $60-64 y$ & $0.56(0.49,0.64)$ & $0.74(0.61,0.89)$ & $0.39(0.34,0.46)$ & $0.54(0.43,0.67)$ \\
\hline $65-69 y$ & $0.55(0.48,0.63)$ & $0.69(0.57,0.85)$ & $0.43(0.37,0.50)$ & $0.56(0.44,0.72)$ \\
\hline $70 y$ or older & $0.43(0.38,0.49)$ & $0.57(0.46,0.70)$ & $0.55(0.48,0.63)$ & $0.68(0.53,0.87)$ \\
\hline \multicolumn{5}{|l|}{ Perceptions of public transport (ref: poor) } \\
\hline fair & $1.15(1.05,1.26)$ & $1.05(0.96,1.16)$ & $1.06(0.94,1.19)$ & $0.92(0.81,1.04)$ \\
\hline very good & $1.23(1.13,1.35)$ & $1.09(1.00,1.20)$ & $1.34(1.20,1.51)$ & $1.10(0.98,1.24)$ \\
\hline excellent & $1.49(1.33,1.66)$ & $1.29(1.15,1.45)$ & $1.90(1.66,2.17)$ & $1.53(1.33,1.76)$ \\
\hline no opinion & $0.71(0.63,0.80)$ & $0.65(0.58,0.74)$ & $0.12(0.09,0.16)$ & $0.10(0.08,0.14)$ \\
\hline missing & $0.56(0.42,0.76)$ & $0.51(0.38,0.69)$ & $0.07(0.03,0.21)$ & $0.06(0.02,0.19)$ \\
\hline \multicolumn{5}{|c|}{ Highest educational qualification (ref: Degree) } \\
\hline Other higher degree & & $0.96(0.87,1.05)$ & & $0.86(0.76,0.98)$ \\
\hline A-level & & $0.95(0.88,1.03)$ & & $0.87(0.78,0.97)$ \\
\hline GCSE & & $1.00(0.92,1.08)$ & & $1.00(0.90,1.11)$ \\
\hline Other qualification & & $1.03(0.93,1.14)$ & & $1.20(1.05,1.37)$ \\
\hline No qualification & & $1.05(0.95,1.17)$ & & $1.51(1.34,1.72)$ \\
\hline Missing & & $1.08(0.84,1.38)$ & & $1.20(0.91,1.60)$ \\
\hline \multicolumn{5}{|l|}{ Economic status (ref: employed) } \\
\hline unemployed & & $1.99(1.75,2.26)$ & & $2.18(1.88,2.52)$ \\
\hline retired & & $1.28(1.14,1.45)$ & & $1.27(1.09,1.49)$ \\
\hline family care & & $1.44(1.28,1.62)$ & & $1.00(0.85,1.17)$ \\
\hline student & & $1.48(1.26,1.75)$ & & $2.23(1.86,2.66)$ \\
\hline sick/disabled & & $1.01(0.83,1.23)$ & & $1.86(1.50,2.30)$ \\
\hline don't know & & $1.18(0.79,1.75)$ & & $0.84(0.47,1.48)$ \\
\hline \multicolumn{5}{|l|}{ Physical functioning (ref: 1 (low)) } \\
\hline 2 & & $1.90(1.66,2.17)$ & & $1.06(0.92,1.22)$ \\
\hline 3 & & $2.30(2.01,2.64)$ & & $1.08(0.93,1.25)$ \\
\hline 4 & & $2.56(2.23,2.93)$ & & $0.96(0.83,1.12)$ \\
\hline 5 (high) & & $2.64(2.30,3.04)$ & & $0.96(0.82,1.13)$ \\
\hline \multicolumn{5}{|l|}{ Urban/rural (ref: urban) } \\
\hline rural & & $0.73(0.68,0.78)$ & & $0.50(0.45,0.55)$ \\
\hline \multicolumn{5}{|l|}{ Random Part } \\
\hline Regional variance (standard error) & $0.048(0.020)$ & $0.036(0.016)$ & $0.359(0.148)$ & $0.294(0.122)$ \\
\hline VPC, MOR & $1.3 \%, 1.23$ & $1.0 \%, 1.20$ & $8.7 \%, 1.77$ & $7.2 \%, 1.68$ \\
\hline Household variance (standard error) & $0.333(0.032)$ & $0.329(0.033)$ & $0.491(0.050)$ & $0.494(0.051)$ \\
\hline VPC, MOR & $9.1 \%, 1.73$ & $9.0 \%, 1.73$ & $11.9 \%, 1.95$ & $12.1 \%, 1.95$ \\
\hline
\end{tabular}

VPC $=$ Variance Partition Coefficient $\mid$ MOR $=$ Median Odds Ratio

https://doi.org/10.1371/journal.pone.0180081.t003 

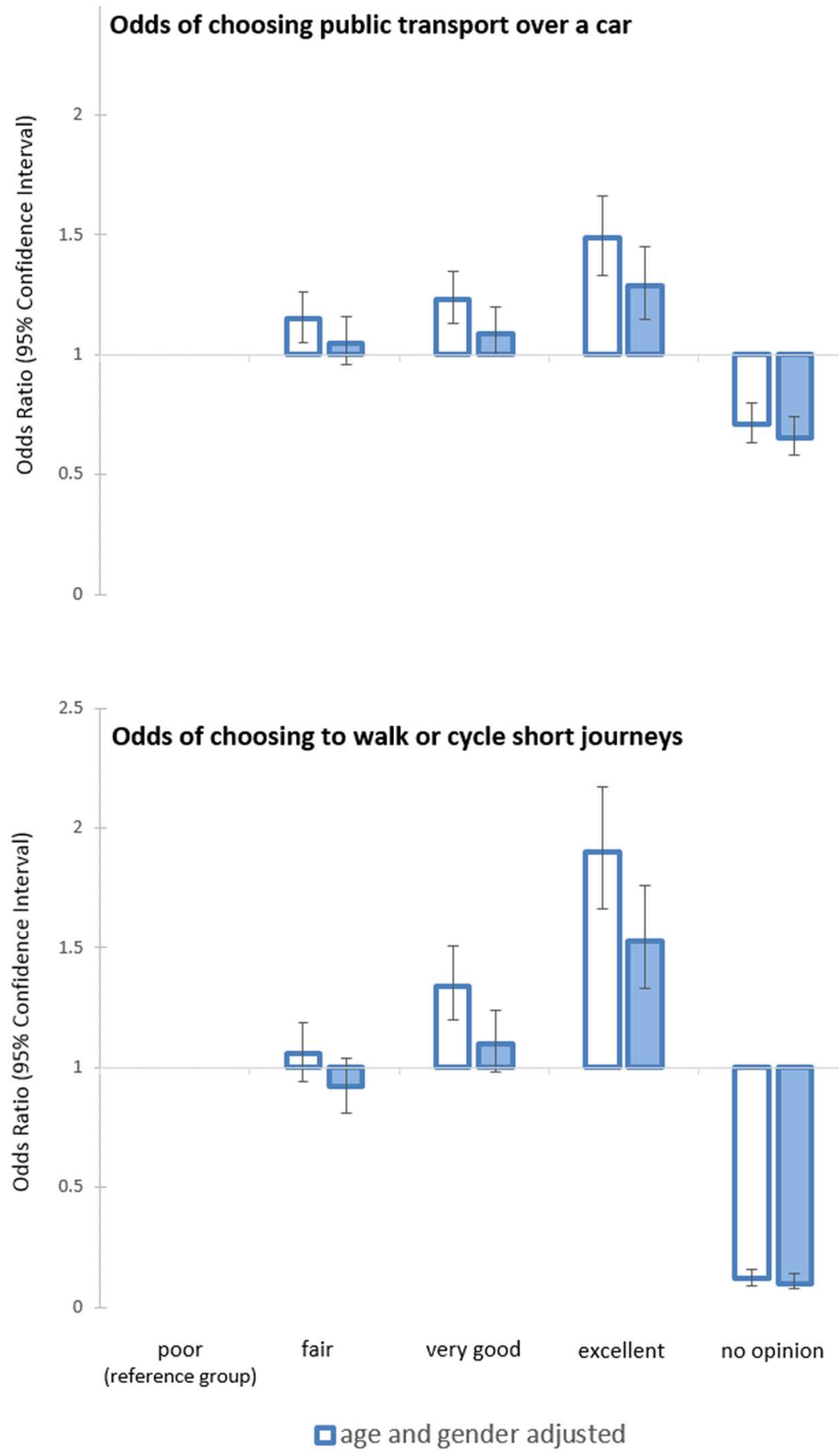

Fig 1. Association between public transport use and perceptions of public transport infrastructure. https://doi.org/10.1371/journal.pone.0180081.g001

$\mathrm{GHQ} \geq 4$ outcome separated frequent public transport users with poor and excellent PPTI. Similarly, a mean difference of 1.13 gap was observed between the same groups for the GHQ$36,-1.4$ for the MCS and -0.5 for the WEMWBS. Poorer mental health was indicative for poorer PPTI also among infrequent users of public transport, though to a demonstrably smaller degree. 
Table 4. Association between mental health, frequency of public transport use and perceptions of public transport infrastructure.

\begin{tabular}{|c|c|c|c|c|}
\hline & GHQ cases $\geq 4$ & GHQ score (linear) & MCS & WEMWBS \\
\hline Fixed Part & OR $(95 \% \mathrm{Cl})$ & Coefficient $(95 \% \mathrm{Cl})$ & Coefficient $(95 \% \mathrm{Cl})$ & Coefficient $(95 \% \mathrm{Cl})$ \\
\hline \multicolumn{5}{|l|}{ Gender (ref: male) } \\
\hline female & $1.41(1.32,1.51)$ & $0.86(0.75,0.98)$ & $-1.42(-1.61,-1.22)$ & $-0.05(-0.14,0.05)$ \\
\hline \multicolumn{5}{|l|}{ Age group (16-19y) } \\
\hline $20-24 y$ & $1.04(0.84,1.28)$ & $0.80(0.41,1.20)$ & $-0.97(-1.66,-0.29)$ & $-0.38(-0.71,-0.05)$ \\
\hline $25-29 y$ & $1.06(0.85,1.31)$ & $1.04(0.63,1.44)$ & $-1.79(-2.50,-1.08)$ & $-0.56(-0.91,-0.22)$ \\
\hline $30-34 y$ & $1.06(0.86,1.30)$ & $1.12(0.73,1.51)$ & $-1.68(-2.37,-1.00)$ & $-0.30(-0.63,0.03)$ \\
\hline $35-39 y$ & $1.01(0.82,1.24)$ & $1.15(0.77,1.54)$ & $-1.45(-2.12,-0.77)$ & $-0.21(-0.54,0.11)$ \\
\hline $40-44 y$ & $1.00(0.81,1.22)$ & $1.26(0.88,1.63)$ & $-1.25(-1.91,-0.59)$ & $-0.11(-0.43,0.21)$ \\
\hline $45-49 y$ & $0.98(0.80,1.20)$ & $1.30(0.92,1.68)$ & $-0.68(-1.34,-0.02)$ & $-0.10(-0.42,0.21)$ \\
\hline $50-54 y$ & $1.01(0.82,1.24)$ & $1.23(0.84,1.61)$ & $-0.49(-1.16,0.17)$ & $0.10(-0.22,0.42)$ \\
\hline $55-59 y$ & $0.77(0.62,0.95)$ & $0.67(0.28,1.06)$ & $0.59(-0.09,1.28)$ & $0.67(0.34,1.00)$ \\
\hline $60-64 y$ & $0.53(0.42,0.67)$ & $-0.06(-0.47,0.36)$ & $1.80(1.07,2.53)$ & $1.29(0.94,1.64)$ \\
\hline $65-69 y$ & $0.43(0.33,0.56)$ & $-0.45(-0.90,0.00)$ & $2.87(2.08,3.65)$ & $1.72(1.33,2.10)$ \\
\hline $70 y$ or older & $0.35(0.27,0.46)$ & $-0.98(-1.43,-0.53)$ & $3.01(2.22,3.80)$ & $1.74(1.36,2.12)$ \\
\hline \multicolumn{5}{|c|}{ PPTI (ref: excellent PPTI, frequent use of public transport) } \\
\hline excellent PPTI, infrequent public transport use & $0.88(0.72,1.07)$ & $-0.21(-0.58,0.15)$ & $0.71(0.06,1.35)$ & $0.28(-0.03,0.59)$ \\
\hline very good PPTI, frequent use of public transport & $0.92(0.76,1.12)$ & $0.08(-0.28,0.43)$ & $0.00(-0.62,0.62)$ & $-0.24(-0.54,0.06)$ \\
\hline very good PPTI, infrequent public transport use & $0.84(0.71,1.00)$ & $-0.05(-0.37,0.27)$ & $0.48(-0.08,1.04)$ & $-0.06(-0.33,0.21)$ \\
\hline fair PPTI, frequent use of public transport & $1.13(0.91,1.41)$ & $0.49(0.07,0.90)$ & $-0.90(-1.63,-0.18)$ & $-0.66(-1.01,-0.31)$ \\
\hline fair PPTI, infrequent public transport use & $0.94(0.79,1.13)$ & $0.32(-0.01,0.65)$ & $-0.17(-0.75,0.41)$ & $-0.46(-0.75,-0.18)$ \\
\hline poor PPTI, frequent use of public transport & $1.46(1.11,1.93)$ & $1.14(0.59,1.69)$ & $-1.44(-2.41,-0.48)$ & $-0.50(-0.97,-0.04)$ \\
\hline poor PPTI, infrequent public transport use & $1.15(0.94,1.40)$ & $0.67(0.31,1.04)$ & $-0.68(-1.33,-0.04)$ & $-0.34(-0.66,-0.03)$ \\
\hline no opinion PPTI, frequent use of public transport & $1.33(0.62,2.84)$ & $-0.60(-2.08,0.87)$ & $0.08(-2.50,2.66)$ & $-0.37(-1.61,0.88)$ \\
\hline no opinion PPTI, infrequent public transport use & $0.84(0.69,1.03)$ & $0.10(-0.26,0.46)$ & $0.48(-0.15,1.11)$ & $-0.02(-0.33,0.29)$ \\
\hline missing & $1.14(0.93,1.40)$ & $0.65(0.27,1.03)$ & $-1.32(-1.99,-0.66)$ & $-0.36(-0.68,-0.04)$ \\
\hline \multicolumn{5}{|l|}{ Highest educational qualification (ref: Degree) } \\
\hline Other higher degree & $1.03(0.92,1.16)$ & $0.17(-0.04,0.37)$ & $-0.39(-0.74,-0.04)$ & $-0.43(-0.60,-0.26)$ \\
\hline A-level & $1.04(0.94,1.15)$ & $0.18(0.01,0.36)$ & $-0.46(-0.77,-0.16)$ & $-0.56(-0.71,-0.41)$ \\
\hline GCSE & $1.06(0.96,1.17)$ & $0.24(0.07,0.41)$ & $-0.62(-0.93,-0.32)$ & $-0.93(-1.08,-0.78)$ \\
\hline Other qualification & $1.11(0.98,1.26)$ & $0.32(0.10,0.55)$ & $-0.73(-1.12,-0.34)$ & $-1.09(-1.27,-0.90)$ \\
\hline No qualification & $1.11(0.98,1.25)$ & $0.36(0.14,0.58)$ & $-1.54(-1.92,-1.15)$ & $-1.12(-1.30,-0.93)$ \\
\hline Missing & $1.03(0.77,1.39)$ & $0.36(-0.17,0.90)$ & $-1.01(-1.95,-0.06)$ & $-0.86(-1.31,-0.40)$ \\
\hline \multicolumn{5}{|l|}{ Economic status (ref: employed) } \\
\hline unemployed & $1.87(1.63,2.15)$ & $1.86(1.57,2.15)$ & $-3.36(-3.86,-2.85)$ & $-1.53(-1.78,-1.29)$ \\
\hline retired & $1.27(1.09,1.49)$ & $0.35(0.09,0.60)$ & $-1.31(-1.76,-0.86)$ & $-0.08(-0.30,0.13)$ \\
\hline family care & $1.30(1.14,1.48)$ & $0.83(0.57,1.10)$ & $-2.03(-2.49,-1.57)$ & $-0.51(-0.73,-0.28)$ \\
\hline student & $1.15(0.95,1.40)$ & $0.62(0.25,0.99)$ & $-0.81(-1.46,-0.16)$ & $0.01(-0.30,0.32)$ \\
\hline sick/disabled & $2.96(2.53,3.47)$ & $4.40(4.05,4.76)$ & $-11.32(-11.94,-10.71)$ & $-3.34(-3.64,-3.04)$ \\
\hline don’t know & $1.68(1.09,2.58)$ & $1.25(0.38,2.11)$ & $-2.50(-4.01,-0.98)$ & $-0.80(-1.53,-0.07)$ \\
\hline \multicolumn{5}{|l|}{ Physical functioning (ref: 1 (low)) } \\
\hline 2 & $0.46(0.41,0.52)$ & $-2.16(-2.39,-1.92)$ & $1.27(0.86,1.67)$ & $0.86(0.66,1.06)$ \\
\hline 3 & $0.27(0.24,0.30)$ & $-3.50(-3.74,-3.26)$ & $1.41(0.99,1.84)$ & $1.57(1.36,1.77)$ \\
\hline 4 & $0.13(0.11,0.15)$ & $-4.81(-5.05,-4.56)$ & $3.44(3.02,3.87)$ & $2.69(2.48,2.90)$ \\
\hline 5 (high) & $0.47(0.41,0.53)$ & $-2.06(-2.31,-1.80)$ & $-4.24(-4.68,-3.79)$ & $1.36(1.15,1.58)$ \\
\hline \multicolumn{5}{|l|}{ Urban/rural (ref: urban) } \\
\hline rural & $0.86(0.79,0.93)$ & $-0.36(-0.51,-0.22)$ & $1.09(0.83,1.34)$ & $0.26(0.14,0.39)$ \\
\hline
\end{tabular}


Table 4. (Continued)

\begin{tabular}{|c|c|c|c|c|}
\hline & GHQ cases $\geq 4$ & GHQ score (linear) & MCS & WEMWBS \\
\hline \multicolumn{5}{|l|}{ Random Part } \\
\hline Regional variance (standard error) & $0.005(0.003)$ & $0.011(0.009)$ & $0.061(0.039)$ & $0.020(0.011)$ \\
\hline Variance Partition Coefficient & $0.1 \%$ & $0.04 \%$ & $0.08 \%$ & $0.11 \%$ \\
\hline Household variance (standard error) & $0.217(0.046)$ & $3.404(0.232)$ & $11.131(0.713)$ & $2.796(0.167)$ \\
\hline Variance Partition Coefficient & $6.2 \%$ & $13.7 \%$ & $14.6 \%$ & $15.7 \%$ \\
\hline Person variance (standard error) & not applicable & $21.403(0.269)$ & $64.941(0.819)$ & $14.967(0.189)$ \\
\hline Variance Partition Coefficient & & $86.2 \%$ & $85.3 \%$ & $84.2 \%$ \\
\hline
\end{tabular}

$\mathrm{GHQ}=$ General Health Questionnaire $\mid$ MCS = SF-12 Mental Health Component $\mid$ WEMWBS = Warwick Edinburgh Mental Well Being Scale

\section{Discussion}

The physical health implications of active travel are increasingly widely known [7-11]. Over short distances, encouraging walking and cycling rather than driving cars is a policy imperative, but over longer distances, promoting the use of public transport is crucial. It is already recognised that investments in walkability and public transport infrastructure are required to promote more equitable, health promoting built environments. Our study supports this general position, with people who more frequently use public transport tending to have more favourable levels of mental health across four contrasting indicators. But, in line with our hypothesis, PPTI modified this association, wherein frequent users of public transport infrastructure perceived to be lower in quality reported poorer mental health across each indicator. Investments in public transport infrastructure must, therefore, also be perceived by the public as safe, easy to use and fit-for-purpose in order to maximise potential co-benefits for the community.

These findings are novel given the paucity of epidemiological research on active travel via public transport that takes into account PPTI. This is somewhat surprising given prior research from the transport literature that emphasises the relationship between perceptions of quality and subsequent use of public transport. Studies in different parts of the world have shown, for example, that services ought to be designed in ways that accommodate the needs of passengers [37]. That those passengers who have to wait for extended periods for buses or trains prefer to do so in conditions that they feel are comfortable, clean, safe, lit, sheltered from the weather and with service staff present [14]. These attributes as well as a reliable service more generally is likely to be particularly important for people who are frequent users of public transport $[13,38]$. As such, it is plausible that for both those who elect to use public transport over a car because it fits their preferences and values and people who have no choice but to use it to get from A to B, perceptions of quality are highly likely to play a potentially powerful role in shaping their wellbeing.

These results also have a bearing for the wider preventive health agenda, since there can be no health without mental health [39]. Provision of public transport infrastructure is a necessary pre-condition to stimulating increases in active travel, but urban planners and other decision makers must take steps to ensure that those investments are attractive to the local populous. Availability does not equate to quality and it is the combination of these elements that are not only crucial to making public transport the default option for longer journeys instead of cars, but also for the protection of mental health for entire populations.

Our study benefits from using large nationally representative data with time-lagged PPTI and confounder variables relative to the mental health and use of public transport indicators, 

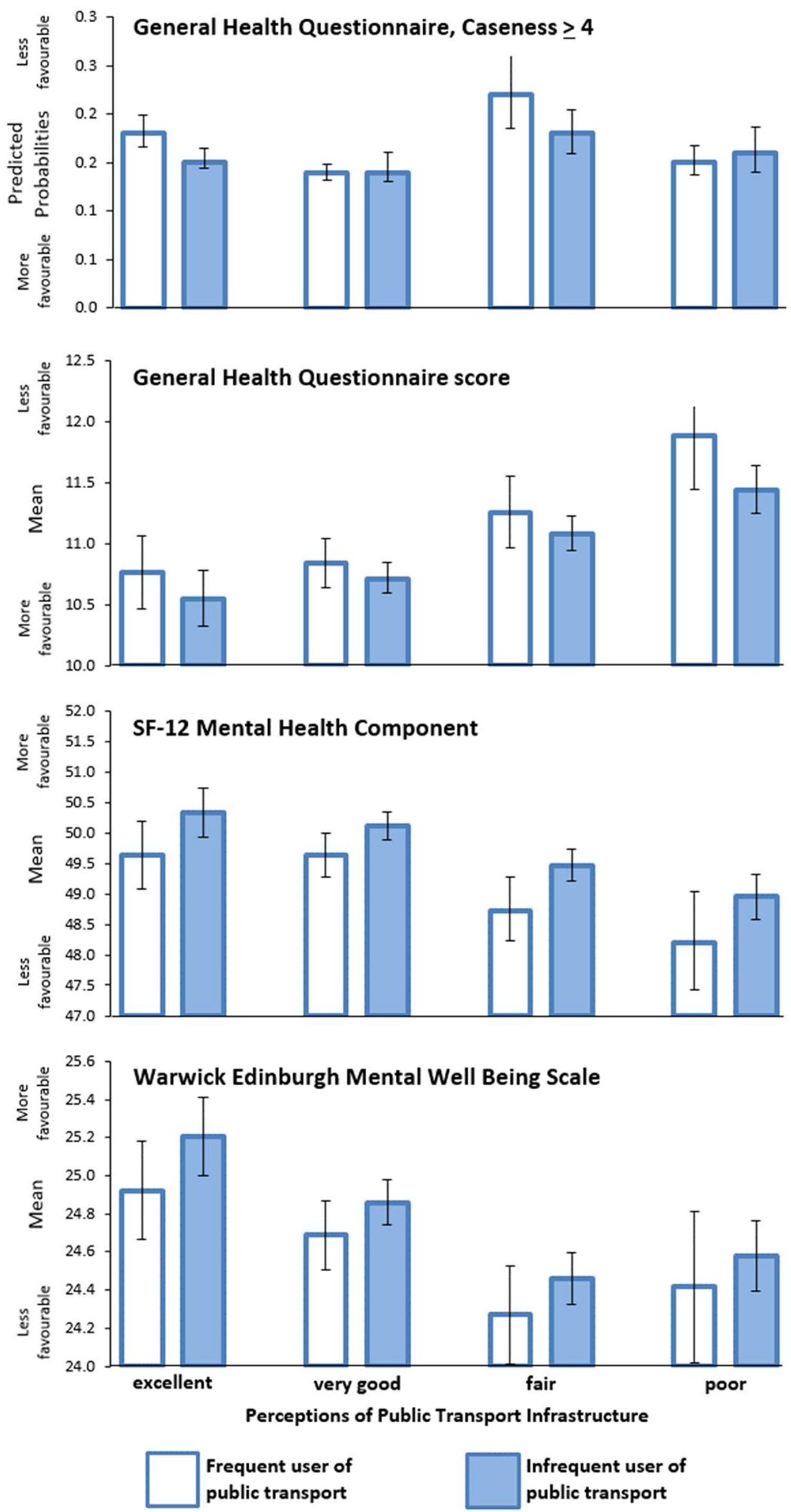

Fig 2. Fully adjusted predictions for the cross-classification of perceptions of public transport and frequency of using of public transport, for 4 contrasting mental health indicators.

https://doi.org/10.1371/journal.pone.0180081.g002 
which helped to avoid bias due to reverse causation. The use of four contrasting mental health indicators is also advantageous, providing opportunities to test the consistency of our results across indicators as diverse as cases of minor psychological morbidity to positive mental wellbeing. Multilevel models also demonstrated the relevance of taking into account household clustering in household surveys, which has previously not always been the case.

A possible limitation of our study includes the reliance upon self-reported data, though it is not known whether objective measurement of public transport quality correlates with the inherently subjective PPTI. Previous research comparing objective and subjective measurement of walkability [40] and green space [41] availability suggests the correlation may not be strong. This is an important area for future interdisciplinary research between epidemiologists and transport researchers as it would indicate the extent that interventions should focus upon physical changes in public transport infrastructure and/or marketing strategies to make existing infrastructure more attractive and well-known.

\section{Acknowledgments}

We thank the participants in Understanding Society: the UK Household Longitudinal Study (UKHLS) for giving up their time to take part in the study and NatCen and their interviewers for undertaking the fieldwork. UKHLS is carried out by the Institute for Social and Economic Research, University of Essex.

\section{Author Contributions}

\section{Conceptualization: XF TAB.}

Data curation: XF TAB.

Formal analysis: XF TAB.

Funding acquisition: $\mathrm{XF}$.

Investigation: $\mathrm{XF} \mathrm{TAB}$.

Methodology: XF TAB.

Project administration: XF TAB.

Resources: XF TAB.

Software: XF TAB.

Supervision: XF TAB.

Validation: XF TAB.

Visualization: XF TAB.

Writing - original draft: XF TAB.

Writing - review \& editing: XF TAB ZF.

\section{References}

1. Foster C, Hillsdon M, Thorogood M, Kaur A, Wedatilake T. Interventions for promoting physical activity. Cochrane database of systematic Reviews. 2005; 1.

2. Commission on the Social Determinants of Health. Closing the gap in a generation: Health equity through action on the social determinants of health: Final Report of the Commission on the Social Determinants of Health. Geneva, Switzerland: WHO; 2008. 
3. Giles-Corti B, Ryan K, Foster S. Increasing density in Australia: maximising the health benefits and minimising harm, to the National Heart Foundation of Australia. Melbourne: National Heart Foundation of Australia; 2012.

4. Rose G. Sick individuals and sick populations. Int J Epidemiol. 1985; 14:32-8. PMID: 3872850

5. Guell C, Panter J, Jones N, Ogilvie D. Towards a differentiated understanding of active travel behaviour: using social theory to explore everyday commuting. Soc Sci Med. 2012; 75(1):233-9. https://doi.org/10. 1016/j.socscimed.2012.01.038 PMID: 22486840

6. Saelens BE, Sallis JF, Frank LD. Environmental correlates of walking and cycling: findings from the transportation, urban design, and planning literatures. Ann Behav Med. 2003; 25(2):80-91. PMID: 12704009

7. Giles-Corti B, Foster S, Shilton T, Falconer R. The co-benefits for health of investing in active transportation. New South Wales public health bulletin. 2010; 21(6):122-7.

8. Jarrett J, Woodcock J, Griffiths UK, Chalabi Z, Edwards P, Roberts I, et al. Effect of increasing active travel in urban England and Wales on costs to the National Health Service. The Lancet. 2012; 379 (9832):2198-205.

9. Rissel CE. Active travel: a climate change mitigation strategy with co-benefits for health. New South Wales public health bulletin. 2009; 20(2):10-3.

10. Sallis JF, Frank LD, Saelens BE, Kraft MK. Active transportation and physical activity: opportunities for collaboration on transportation and public health research. Transportation Research Part A: Policy and Practice. 2004; 38(4):249-68.

11. Saunders LE, Green JM, Petticrew MP, Steinbach R, Roberts $H$. What are the health benefits of active travel? A systematic review of trials and cohort studies. PLoS One. 2013; 8(8):e69912. https://doi.org/ 10.1371/journal.pone.0069912 PMID: 23967064

12. Pont K, Ziviani J, Wadley D, Bennett S, Abbott R. Environmental correlates of children's active transportation: a systematic literature review. Health \& place. 2009; 15(3):849-62.

13. Dell'Olio L, Ibeas $A$, Cecin $P$. The quality of service desired by public transport users. Transport Policy. 2011; 18(1):217-27.

14. Paulley N, Balcombe R, Mackett R, Titheridge $H$, Preston J, Wardman $M$, et al. The demand for public transport: The effects of fares, quality of service, income and car ownership. Transport Policy. 2006; 13 (4):295-306.

15. Sapolsky RM. The influence of social hierarchy on primate health. Science. 2005; 308(5722):648-52. https://doi.org/10.1126/science.1106477 PMID: 15860617

16. Marmot MG. Status syndrome. JAMA: the journal of the American Medical Association. 2006; 295 (11):1304-7. https://doi.org/10.1001/jama.295.11.1304 PMID: 16537740

17. Seeman TE, McEwen BS, Rowe JW, Singer BH. Allostatic load as a marker of cumulative biological risk: MacArthur studies of successful aging. Proceedings of the National Academy of Sciences. 2001; 98(8):4770-5.

18. Humphreys DK, Goodman A, Ogilvie D. Associations between active commuting and physical and mental wellbeing. Prev Med. 2013; 57(2):135-9. https://doi.org/10.1016/j.ypmed.2013.04.008 PMID: 23618913

19. Ellaway A, Macintyre S, Hiscock R, Kearns A. In the driving seat: psychosocial benefits from private motor vehicle transport compared to public transport. Transportation Research Part F: Traffic Psychology and Behaviour. 2003; 6(3):217-31.

20. Le Vine S, Jones P. On the move: making sense of car and train travel trends in Britain. 2012.

21. Preston J, Robins D. Evaluating the long term impacts of transport policy: The case of passenger rail privatisation. Research in Transportation Economics. 2013; 39(1):14-20.

22. Tyrrall D. The UK railway privatisation: failing to succeed? Economic Affairs. 2004; 24(3):32-8.

23. Buck N, McFall S. Understanding Society: design overview. Longitudinal and Life Course Studies. 2011; 3(1):5-17.

24. Knies G. The UK Household Longitudinal Study Waves 1-4, User Manual. Colchester, Essex: Institute for Social and Economic Research, University of Essex; 2014.

25. Goldberg DP. The detection of psychiatric illness by questionnaire. London: Oxford University Press; 1972.

26. Goldberg D, Williams P. A user's guide to the General Health Questionnaire. Windsor: NFER-nelson; 1988.

27. Goldberg D, Oldehinkel T, Ormel J. Why GHQ threshold varies from one place to another. Psychol Med. 1998; 28(4):915-21. PMID: 9723146 
28. Ware JE Jr, Sherbourne CD. The MOS 36-item short-form health survey (SF-36): I. Conceptual framework and item selection. Med Care. 1992:473-83. PMID: 1593914

29. Ware JE Jr, Kosinski M, Keller SD. A 12-Item Short-Form Health Survey: construction of scales and preliminary tests of reliability and validity. Med Care. 1996; 34(3):220-33. PMID: 8628042

30. Tennant R, Hiller L, Fishwick R, Platt S, Joseph S, Weich S, et al. The Warwick-Edinburgh mental wellbeing scale (WEMWBS): development and UK validation. Health and Quality of life Outcomes. 2007; 5 (1):63.

31. Stewart-Brown SL, Platt S, Tennant A, Maheswaran H, Parkinson J, Weich S, et al. The Warwick-Edinburgh Mental Well-being Scale (WEMWBS): a valid and reliable tool for measuring mental well-being in diverse populations and projects. J Epidemiol Community Health. 2011; 65(Suppl 2):A38-A9.

32. Merlo J, Chaix B, Ohlsson H, Beckman A, Johnell K, Hjerpe P, et al. A brief conceptual tutorial of multilevel analysis in social epidemiology: using measures of clustering in multilevel logistic regression to investigate contextual phenomena. J Epidemiol Community Health. 2006; 60(4):290-7. https://doi.org/ 10.1136/jech.2004.029454 PMID: 16537344

33. Rasbash J, Browne W, Goldstein H, Yang M, Plewis I, Healy M, et al. A user's guide to MLwiN. London: Institute of Education. 2000;286.

34. Hirsch JA, Diez Roux AV, Moore KA, Evenson KR, Rodriguez DA. Change in walking and body mass index following residential relocation: the multi-ethnic study of atherosclerosis. Am J Public Health 2014; 104(3):e49-e56. https://doi.org/10.2105/AJPH.2013.301773 PMID: 24432935

35. Fryers $\mathrm{T}$, Melzer $\mathrm{D}$, Jenkins $\mathrm{R}$. Social inequalities and the common mental disorders. Soc Psychiatry Psychiatr Epidemiol. 2003; 38(5):229-37. https://doi.org/10.1007/s00127-003-0627-2 PMID: 12719837

36. Paluska SA, Schwenk TL. Physical activity and mental health. Sports Med. 2000; 29(3):167-80. PMID: 10739267

37. Beirão G, Cabral JS. Understanding attitudes towards public transport and private car: A qualitative study. Transport policy. 2007; 14(6):478-89.

38. Dell'Olio L, Ibeas A, Cecín P. Modelling user perception of bus transit quality. Transport Policy. 2010; 17(6):388-97.

39. Prince M, Patel V, Saxena S, Maj M, Maselko J, Phillips MR, et al. No health without mental health. The lancet. 2007; 370(9590):859-77.

40. Gebel K, Bauman AE, Sugiyama T, Owen N. Mismatch between perceived and objectively assessed neighborhood walkability attributes: prospective relationships with walking and weight gain. Health \& place. $2011 ; 17(2): 519-24$.

41. Macintyre S, Macdonald L, Ellaway A. Lack of agreement between measured and self-reported distance from public green parks in Glasgow, Scotland. International Journal of Behavioral Nutrition and Physical Activity. 2008; 5(1):26. 